\title{
Plant Growth Promoting Rhizobacteria (PGPR) Regulated Phyto and Microbial Beneficial Protein Interactions
}

https://doi.org/10.1515/biol-2020-0008

Received November 8, 2019; accepted November 22, 2019

\begin{abstract}
Plant Growth Promoting Rhizobacteria (PGPR) influence plants' physiological characteristics, metabolites, pathways and proteins via alteration of corresponding gene expression. In the current study, a total of 42 upregulated uncharacterized sorghum bicolor root proteins influenced by PGPR were subjected to different analyses: phylogenetic tree, protein functional network, sequences similarity network (SSN), Genome Neighborhood Network (GNN) and motif analysis. The screen for homologous bacterial proteins to uncover associated protein families and similar proteins in non-PGPRs was identified. The sorghum roots' uncharacterized protein sequences analysis indicated the existence of two protein categories, the first being related to phytobeneficial protein family associated with DNA regulation such as Sulfatase, FGGY_C, Phosphodiesterase or stress tolerance such as HSP70. The second is associated with bacterial transcriptional regulators such as FtsZ, MreB_Mbl and DNA-binding transcriptional regulators, as well as the AcrR family, which existed in PGPR and non PGPR. Therefore, Plant Growth-Promoting Rhizobacteria (PGPR) regulated phytobeneficial traits through reciprocal protein stimulation via microbe plant interactions, both during and post colonization.
\end{abstract}

Keywords: Plant Growth promoting rhizobacteria, Sorghum, phylogenetic analysis, protein family, microbe interactions

\section{Introduction}

The successful use of Plant-Growth-Promoting Rhizobacteria (PGPR) in agriculture is correlated with reciprocal gene regulation between bacteria and plants during plant colonization. This gene regulation exerts phytobeneficial results on biomass, nutrient uptake and metabolite upregulation [1-5] on proteins and biological pathways [6], as well as on gene expression [7]. In the current study, the PGPR-Pseudomonas sp. TLC 6-6.5-4as previously used [1] was applied to two-day-old sorghum seedlings to check the function of upregulated, uncharacterized root proteins three months after PGPR colonization. The study's main objective was to reveal the function of uncharacterized sorghum bicolor proteins in roots treated with PGPR. In order to identify the functions of these proteins, several steps were conducted. Sorghum root uncharacterized protein sequences were used in a Blast search for homologous protein sequences in bacteria, since they were unidentified in plant protein sequences. In total, 564 proteins were subjected to several analyses to determine their possible role in phytobeneficial traits. The analyses included the following: sequence similarity network (SSN), Genome Neighborhood Network (GNN), functional network and motif identification. The sorghum roots' uncharacterized protein analysis revealed the existence of protein families belonging to plant, PlantGrowth-Promoting Rhizobacteria (PGPR) and Non-PlantGrowth-Promoting Rhizobacteria (non-PGPR), thus emphasizing the role of microbe plant interactions during and post colonization.

\section{Material and methods}

\subsection{Plant growth conditions and treatments}

*Corresponding author: Faten Dhawi, Agricultural Biotechnology
Department, College of Agriculture and Food Science, King Faisal University, PO Box 400 Al Hofuf, Al-Ahsa 31982, Saudi Arabia, E-mail: dr.faten.dhawi@gmail.com; falmuhanna@kfu.edu.sa
Two-day-old germinated seedlings of sorghum bicolor were inoculated with PGPR Pseudomonas sp. TLC 6-6.5-4 
[1] for two hours, after which they were planted in $600 \mathrm{~g}$ of pasteurized mixed soil consisting of coarse sand and loam (1:1). Pseudomonas sp. TLC 6-6.5-4 was grown on Luria-Bertani (LB) agar for $48 \mathrm{~h}$, harvested, suspended and dissolved in $120 \mathrm{ml}$ of $0.85 \% \mathrm{NaCl}$ solution to reach $10-8 \mathrm{cfu} / \mathrm{mL}$, which was used for the sorghum seedling inoculation and was sprayed on the soil surface. The growth conditions for sorghum in a greenhouse were 14 hours of light, at $30^{\circ} \mathrm{C}$ and $65 \%$ humidity. The experiment consisted of seven sorghum bicolor treated with PGPR Pseudomonas sp. TLC 6-6.5-4- and seven untreated sorghum bicolor as a control. Sorghum bicolor plants were harvested after 90 days for root protein analyses. Roots were washed with distilled water to remove soil then frozen in liquid nitrogen. Protein was extracted from frozen roots; three samples for each group followed the procedure described in Dhawi et al. [6] and modified from Fukao et al. [8].

Three protein samples for each group (treated and control) were digested with trypsin and then analyzed using LC-MS/MS on a Waters/Micromass AB QSTAR Elite (Waters nano ACQUITY ultra high-pressure liquid chromatograph UPLC) for peptide separation. The detected peptides were quantified and normalized to obtain the abundance of each sample according to Progenesis QI (http://www.nonlinear.com/progenesis/qi/ v2.0/faq/hownormalisation-works.aspx). Protein levels of two folds and above in comparison to the control were considered significant change to be consider in further analysis.

\subsection{Sorghum root uncharacterized protein processing}

The sequences of the 42 uncharacterized sorghum root proteins (Table 1) were used to search for homologous bacterial proteins using PSI Blast (https://blast.ncbi.nlm. nih.gov/Blast.cgi?PAGE=Proteins), which resulted in 830 protein sequences. These sequences were filtered using Cluster Database at High Identity with Tolerance (CD-HIT) Suite: Biological Sequence Clustering and Comparison (http://weizhongli-lab.org/cdhit_suite/cgi-bin/index. cgi) with a $90 \%$ identity cut-off. The $90 \%$ cut-off step resulted in 564 protein sequences for inclusion in the multiple sequence alignment (MSA). MSA was created by using the MEGA7 (Molecular Evolutionary Genetics Analysis) software version 6 [9]. The phylogenetic tree was established in MEGA 6 and aligned via the Muscle program for 564 proteins, 42 of which were sorghum root uncharacterized upregulated proteins. Protein sequences
Table 1. Uncharacterized sorghum proteins fold changes influenced by Pseudomonas $s p$. in comparison with control

\begin{tabular}{|c|c|}
\hline Protein ID & Fold change \\
\hline C5YZ77 & 5.5 \\
\hline C5XHS5 & 4.8 \\
\hline C5X5F0 & 4.7 \\
\hline C $5 \times \mathrm{XV} 25$ & 4.1 \\
\hline $\mathrm{C} 5 \mathrm{XFI} 2$ & 3.9 \\
\hline C5YUN2 & 3.7 \\
\hline C5XGS9 & 3.5 \\
\hline C5YPP6 & 3.2 \\
\hline C5XLV5 & 2.9 \\
\hline C5Y9B5 & 2.5 \\
\hline C5WXA4 & 2.3 \\
\hline C5YPX8 & 2.3 \\
\hline C5XC95 & 2.3 \\
\hline C5X4M5 & 2.3 \\
\hline C5WXN2 & 2.1 \\
\hline C5YBP8 & 1.9 \\
\hline C5Y921 & 1.9 \\
\hline C5XYX0 & 1.9 \\
\hline C5YC51 & 1.8 \\
\hline C5YU58 & 1.8 \\
\hline C5YR14 & 1.7 \\
\hline C5Y6L4 & 1.7 \\
\hline C5XG11 & 1.7 \\
\hline C5YAG2 & 1.7 \\
\hline C5YYY8 & 1.7 \\
\hline C5YDI2 & 1.7 \\
\hline $\mathrm{C} 5 \mathrm{XCl9}$ & 1.7 \\
\hline C5YIX7 & 1.7 \\
\hline C5YYX1 & 1.7 \\
\hline C5WT78 & 1.6 \\
\hline C5WXD7 & 1.6 \\
\hline C5XG44 & 1.6 \\
\hline C5YXQ9 & 1.6 \\
\hline C5YEW3 & 1.6 \\
\hline C5Z7E8 & 1.6 \\
\hline C5Z1X3 & 1.6 \\
\hline C5YI64 & 1.6 \\
\hline C5WRP7 & 1.5 \\
\hline C5XHR8 & 1.5 \\
\hline C5Y651 & 1.5 \\
\hline C5XWM5 & 1.5 \\
\hline C5Z8A9 & 1.5 \\
\hline
\end{tabular}


were aligned using neighbor-joining [10] and UPGMA (Unweighted Pair Group Method), with a bootstrap method of 500 replications for a phylogeny test. The interactive tree of life (iTol) online web tool (http://itol.embl.deg) was then used to display and modify the tree colors [11].

\section{Protein functional network and family assignment}

A combination of different approaches was used to assign homologous proteins to protein families (Pfam) with a putative function. In total, 564 protein sequences consisting of sorghum root uncharacterized protein and similar bacterial protein sequences were used to determine functions via the genomic, protein and gene interactions through the sequence similarity network (SSN) and Genome Neighborhood Network (GNN) approach, respectively. The GNN approach was performed using the Enzyme Similarity Tool-Enzyme Function Initiative (EFI-EST) https://efi.igb.illinois.edu/efi-est/ [12]. In order to use EFI-EST, the 564 proteins were converted to UniProt Id, resulting in 554 identified proteins in the UniProt database and 10 unidentified bacterial proteins. The EFI-EST website was used to generate a sequence similarity network (SSN), and the resulting files were visualized in Cytoscape software [13]. Sorghum protein sequences were screened for motifs using the MEME (Multiple EM for Motif Elicitation) tool (http://memesuite.org/tools/meme) [14], with a maximum number of eight motifs for the detection level and an E-value equal to or less than 0.05 . Further analyses were performed for protein families using STRING Consortium 2019 (https:// string-db.org/) [15].

\section{Results}

\subsection{Phylogenetic tree analysis}

The results showed 42 sorghum proteins according to UniProt (http://www.uniprot.org/), which were upregulated from 2-5-fold when compared to the control group. The 42 upregulated sorghum proteins have an unknown function; therefore, several analyses and different approaches were used to identify the function and check for similarity of these uncharacterized proteins with bacterial proteins via an NCBI search (https://blast. ncbi.nlm.nih.gov/Blast.cgi).

The phylogenetic tree of 564 protein sequences included 42 uncharacterized sorghum proteins and similar bacterial proteins aligned using Multiple Sequence alignments (MUSCLE) with a bootstrap of 500. The resulting unrooted phylogenetic tree consisted of

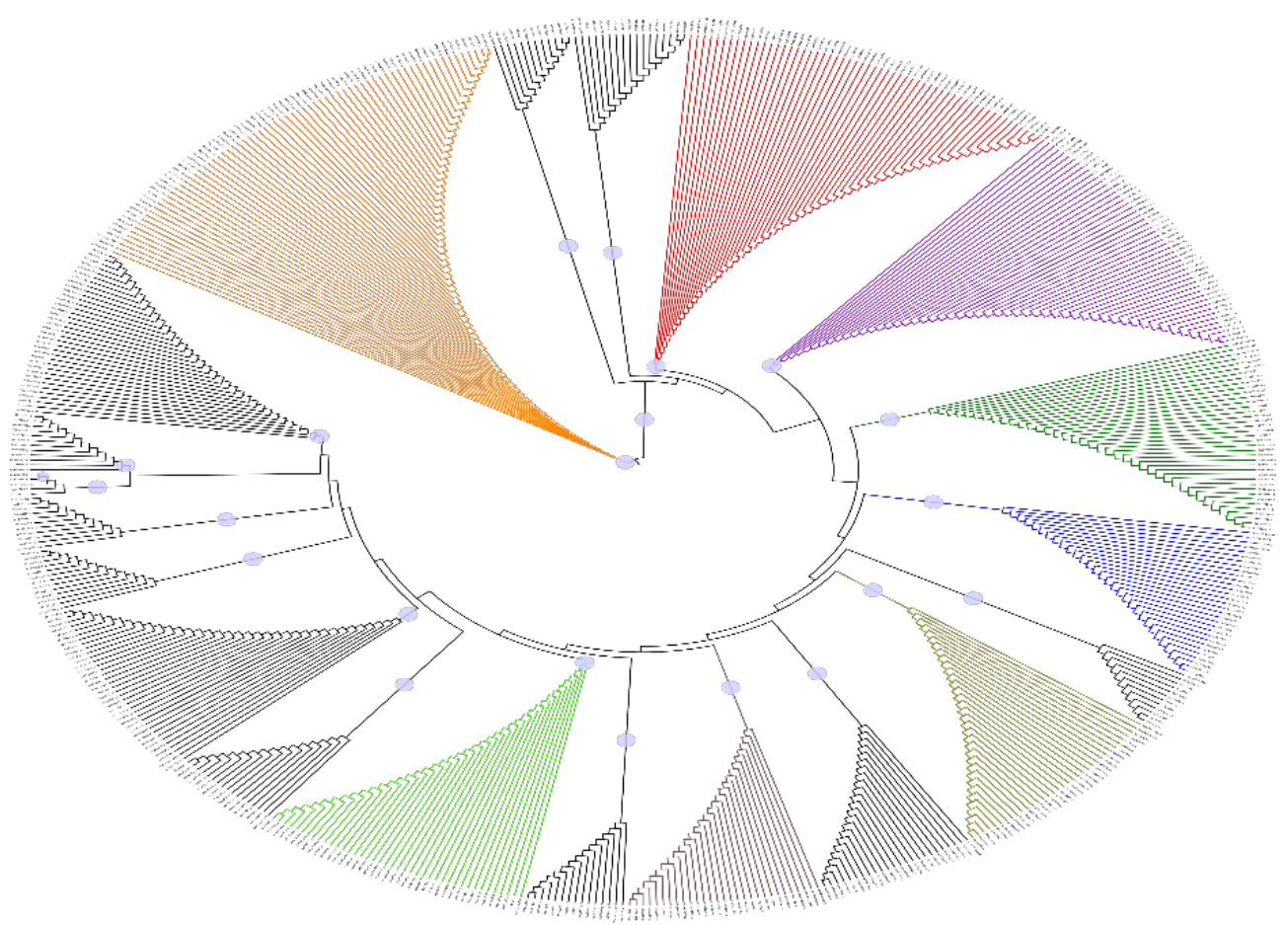

Figure 1. The phylogenetic tree of uncharacterized sorghum proteins and similar bacterial proteins. The unrooted neighbor-joining phylogenetic tree constructed based on 564 multiple proteins sequence alignment using MUSCLE with 500 bootstrap replicates. 
20 nodes, in which sorghum uncharacterized protein sequences were clustered in five of them (Figure 1). The motif analysis of these sorghum and bacterial protein sequences clustered in the same nodes identified eight similar protein family domains represented by motif logos in Figure 2, namely, Sulfatase, CARBOHYDRATE KINASES (FGGY _C), Phosphodiest, Cell division protein FtsA, GSDH, MreB_Mbl, StbA and HSP70.

\subsection{Sequence similarity network (SSN)}

The SSN used to analyze 554 sequences consisted of 42 uncharacterized sorghum protein sequences and 507 bacterial protein sequences. These sequences were obtained by PSI-BLAST at $\mathrm{E}$ values of 10-15; the sequences were clustered based on their similarities in seven clusters. The SSN clusters containing sorghum proteins were cluster 1 (61 protein sequences), cluster 3 (23 sequences), cluster 5 (17 protein sequences), cluster 14 (seven protein sequences), cluster 16 (five protein sequences), cluster 19 (four protein sequences) and cluster 24 (three protein families) (Figure 3). Some proteins were separated with no clusters due to a different $E$ value score. These SSN results were used to establish a Genome Neighborhood Network (GNN).

\subsection{Genome Neighborhood Networks (GNN), motif screening and functional partenrs}

The SNN results were used to build GNNs. The output of the GNN in the Hub-Nodes format showed the number of Pfam gene neighbors that were found in each cluster of sequences. The SNN revealed that uncharacterized protein sequences of sorghum were clustered in seven nodes. The nodes included 1, 3, 5, 14, 16, 19 and 24 protein families, arranged based on their genomic context (Fig.4). Nodes shared 86 protein families (See appendix Table1). On the other hand, the motif scan analysis of sorghum root proteins identified four main protein families: HSP70, MreB_Mbl, StbA and FtsA (Figure 4). The functional protein association network analysis using STRING 2019 predicted nine functional partners. These functional partners were N-acetyltransferase, the GNAT superfamily (includes histone acetyltransferase HPA2), DNA-binding transcriptional regulator, AcrR family; MFS family permease, SAM-dependent methyltransferase, DNA-binding transcriptional regulator, ArsR family, cAMP-binding domain of CRP or a regulatory subunit of cAMP-dependent protein kinases, glycosyltransferase involved in cell wall biosynthesis, signal transduction histidine kinase, and sugar kinase of the NBD/HSP70 family containing an N-terminal HTH domain (Figure 5).

Figure 2. The phylogenetic tree of uncharacterized sorghum proteins and similar bacterial proteins. The unrooted neighbor-joining phylogenetic tree constructed based on the sequence alignment of 564 multiple proteins using Multiple Sequence alignments (MUSCLE) with 500 bootstrap replicates. 


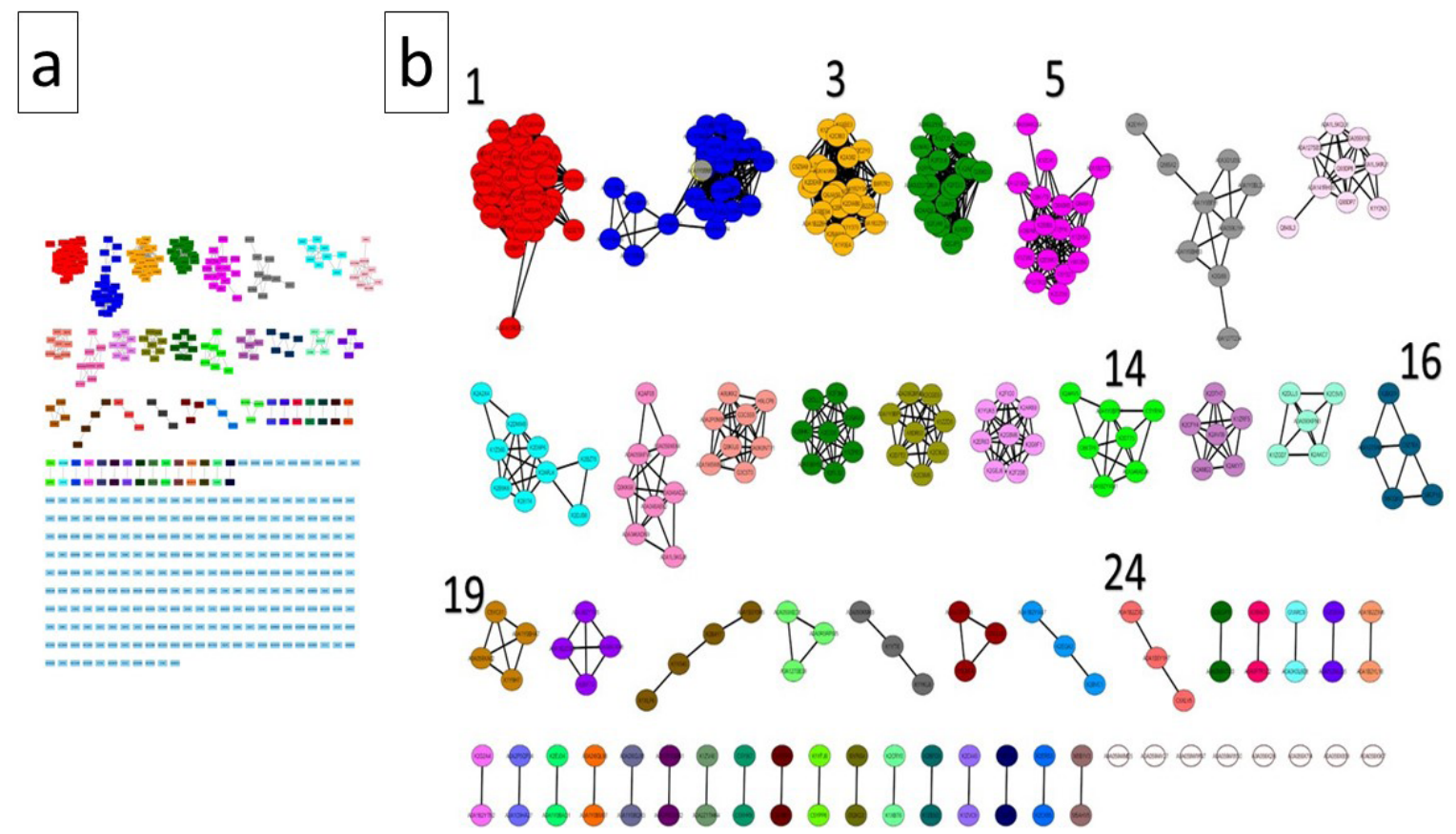

Figure 3. a) Sequence similarity network of uncharacterized sorghum and similar bacterial proteins consisting of 564 sequences at an $E$ value of $10^{-15}$. b) Sorghum uncharacterized proteins clustered in seven groups, with different protein nodes. Each node (circle) represents proteins with similar sequences; the lines indicate the pairwise relationship between sequences. Proteins that have no sorghum sequences appeared as separated and are not labelled.

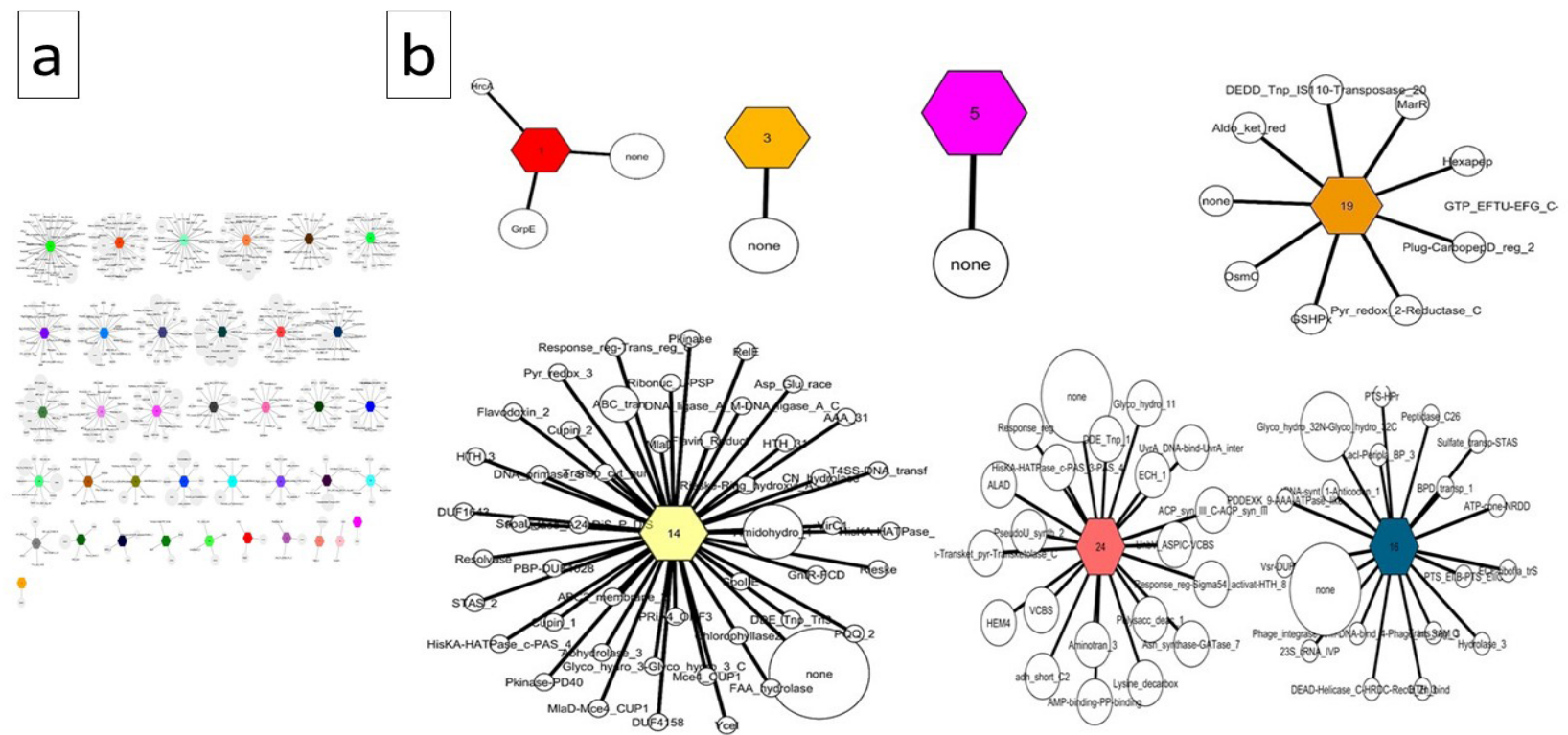

Figure 4. a) Genome Neighborhood Networks (GNNs) in the Hub-Nodes format for 564 protein sequences. b) The hexagon shapes (hubs) represent sequence similarity network (SSN) clusters, and the other shapes (nodes) represent the Pfam genome neighbors. Seven node clusters are indicated with 94 genes. The names of nodes are the short name of the protein family (Pfam). 


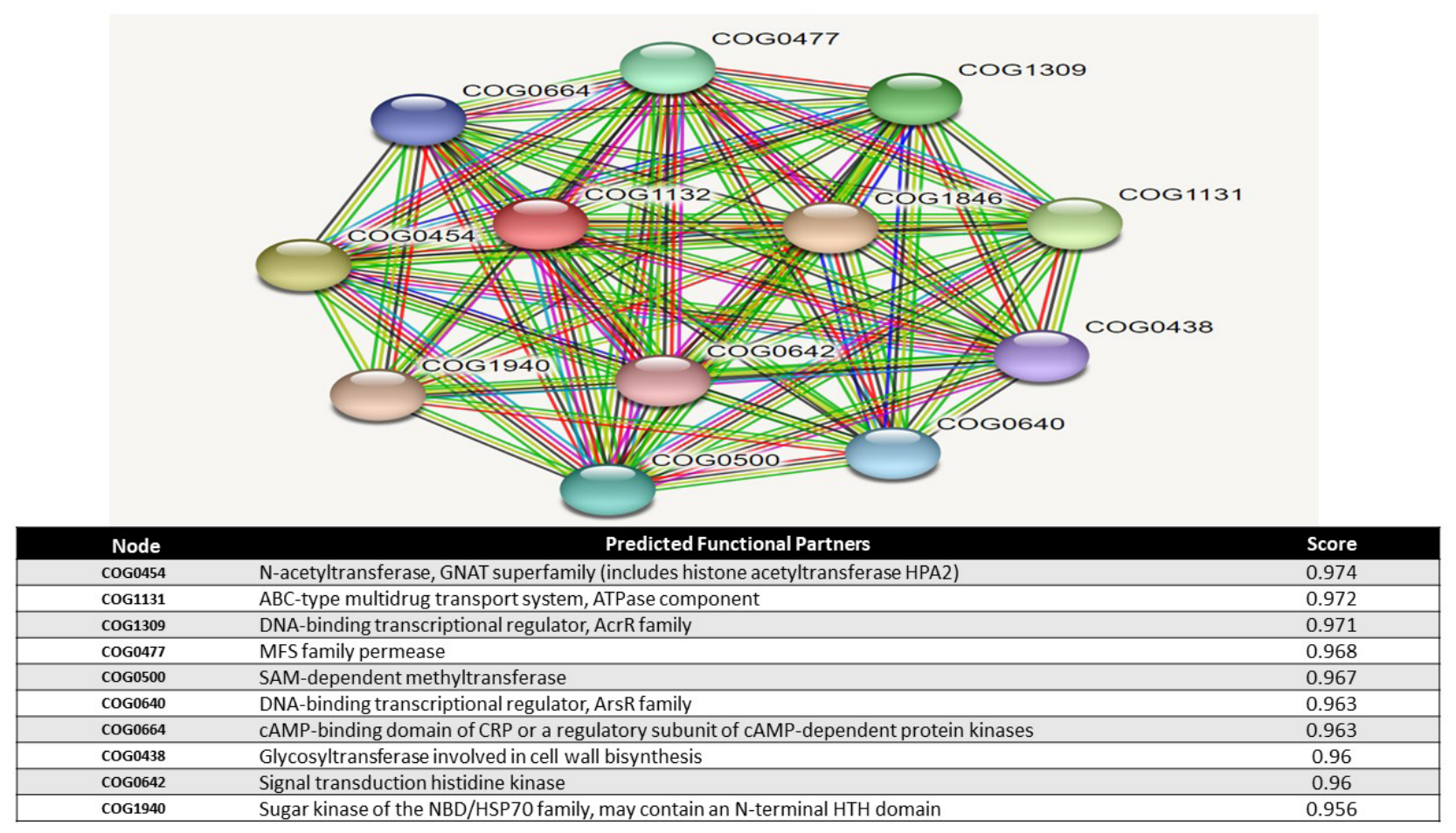

Figure 5. The functional protein association networks for 86 protein families resulting from GNN using the STRING Consortium 2019 functional protein association networks (https://string-db.org/) Szklarczyk et al., [15].

\section{Discussion}

The phylogenetic tree analysis of the 20 nodes identified that five nodes were sorghum proteins clustered with similar bacterial proteins. The motif analysis of these sorghum and bacterial protein sequences clustered in the same nodes revealed six protein family domains (Sulfatase, CARBOHYDRATE KINASES (FGGY _C), Phosphodiesterase, FtsZ, MreB_Mbl and HSP70). Some members of this protein family have no eukaryotic existence, but this might have remained in root cells following plant treatment and may play an intrinsic role. This resulted in phytobeneficial traits such as tolerance to salinity [16], heavy metal [1, 2], and drought [17], or increased plant biomass under normal environmental conditions [18]. Similarly, the results of SNN, GNN and STRING protein functional networks of sorghum root proteins identified the main protein families: HSP70, MreB_Mbl, StbA and FtsA. Therefore, protein analysis revealed the existence of protein families belonging to plant, Plant-Growth-Promoting Rhizobacteria (PGPR) and Non-Plant-Growth-Promoting Rhizobacteria (non-PGPR), thus emphasizing the reciprocal benefit synchronized between plant and bacteria during and post colonization.

\subsection{Phytobeneficial proteins induced by PGPR:}

Plant-Growth-Promoting Rhizobacteria (PGPR) induced several phytobeneficial and desired traits such as an increase in production or tolerance during biotic or abiotic stress [19]. The beneficial effect is associated with an increase in gene expression and certain protein families such as sulfatase substrates, which are involved in hormone regulation, cellular degradation and the modulation of signaling pathways. The increase of element compositions post plant inoculation with PGPR might be explained by sulfatase's ability to cycle environmental sulfur via degradation or cellular remodeling [20]. The increase of biomass in plants is associated with the increased sugars and carbohydrates represented in this study due to an increase in the Carbohydrate kinases (FGGY _C) protein family, which is involved in bacterial signaling molecules [21]. In addition, FGGY carbohydrate kinases contributed plant adenosine and thriphosphate (ATP)-dependent phosphorylation of nine distinct sugar enzymes. These enzymes include L-fucolokinase, gluconokinase, glycerol kinase, xylulokinase, D-ribulose kinase and L-xylulose kinase [22]. In the current 
study, we identified two distinct protein families, Phosphodiesterase and Heat S hock Protein 70 (Hsp70), that are known to contribute to high plant tolerance. Phosphodiesterase is involved in independent pathways of DNA-protein crosslink repair in plants [23]. However, Heat Shock Protein 70 (Hsp70) is an evolutionarily conserved family of proteins that is typically localized in the cytoplasm and distributed in the intermembrane space of chloroplasts. Hsp70 plays a crucial role in protein biogenesis, protection during stress, assistance in protein translocation [24], protection against several stress types, and the maintenance of cellular homeostasis [25]. The upregulation of several sorghum proteins associated with an increase in N-acetyltransferase, the GNAT superfamily (including histone acetyltransferase HPA2) was observed in the current study, and this is believed to be involved in the regulation of the transcription of many genes [26].

\subsection{Rhizobacterial transcriptional regulators}

Several protein families related to bacteria were found in sorghum roots post plant inoculation that work as functional gene regulators. Some of these proteins were identified in a previous study and are known to contribute to phytobeneficial traits related to PGPR [7]. Other proteins are known as bacterial proteins induced by plant root exudates and participate in the bacterial gene regulation of PGPR, such as Bacillus atrophaeus [27]. Some of the predicted functionally related bacterial protein families are known bacterial transcriptional regulators, such as FtsZ, MreB_Mbl, DNA-binding transcriptional regulator and the AcrR family, as shown by the results of the current study. These functionally related protein families are MFS family permease, SAM-dependent methyltransferase, Sadenosyl methionine (SAM), DNA-binding transcriptional regulator, ArsR family transcriptional regulator, the cAMP-binding domain of CRP or a regulatory subunit of cAMP-dependent protein kinases, DNA-binding and transcription regulation, glycosyltransferase, and histidine kinases (HKs).

The family of FtsZ is a bacterial membrane tubulinrelated cell division protein [28]. $\mathrm{MreB} / \mathrm{Mbl}$ is a genecoding protein related to the bacterial protein FtsZ cell membrane protein [29]. Additionally, the analysis identified possible roles of the bacteria-DNA-binding transcriptional regulators known as the AcrR family [30] and the association of amino acid transfer proteins known as MFS family permease [31]. Thus, we emphasized the role of plant root exudates in enhancing bacterial growth activity via the activation of functional partner proteins such as SAM-dependent methyltransferase S-adenosyl methionine (SAM), which is known to transfer the methyl group to DNA [32]. The DNA-binding transcriptional regulator and ArsR family transcriptional regulator are involved in lipid metabolism regulation [33], cAMPdependent protein kinase DNA-binding and transcription regulation [34], while glycosyltransferase is involved in cell wall biosynthesis [35], and histidine kinases (HKs) are known to function in bacterial signal transduction [36].

\section{Conclusion}

Plant Growth Promoting Rhizobacteria (PGPR) regulated phytobeneficial traits by reciprocal protein stimulation via microbe-plant interactions during and post colonization. Furthermore, plant root exudates stimulated bacterial gene regulators associated with bacterial signaling, DNAbinding transcriptional regulators, and cell growth.

Data Availability: Data that are supplementary to the manuscript will be available by request.

Acknowledgements: We express deep gratitude to Dhawi and Safia for their great help during the process of research development. This study was self-funded in fully supported by the author.

Conflict of interest: Authors state no conflict of interest.

\section{References}

[1] Dhawi F, Datta R, Ramakrishna W. Mycorrhiza and PGPB modulate maize biomass, nutrient uptake and metabolic pathways in maize grown in mining-impacted soil. Plant Physiology and Biochemistry 2015; 97, 390-399.

[2] Dhawi F, Datta R, Ramakrishna W. Mycorrhiza and heavy metal resistant bacteria enhance growth, nutrient uptake and alter metabolic profile of sorghum grown in marginal soil. Chemosphere 2016; 157, 33-41.

[3] Dhawi F, Hess A. Plant Growth-Prompting Bacteria Influenced Metabolites of Zea mays var. amylacea and Pennisetum americanum p. in a Species-Specific Manner. Advances in Biological Chemistry 2017a; 7(05), 161.

[4] Dhawi F, Hess A. Poor-Soil Rhizosphere Enriched with Different Microbial Activities Influence the Availability of Base Elements. Open Journal of Ecology 2017b; 7(08), 495.

[5] Dhawi F, Datta R, Ramakrishna W. Proteomics provides insights into biological pathways altered by plant growth promoting bacteria and arbuscular mycorrhiza in sorghum grown in marginal soil. Biochimica et Biophysica Acta (BBA)-Proteins and Proteomics 2017; 1865(2), 243-251.

[6] Dhawi F, Datta R, Ramakrishna W. Metabolomics, biomass and lignocellulosic total sugars analysis in foxtail millet (Setaria 
italica) inoculated with different combinations of plant growth promoting bacteria and mycorrhiza. Communication in Plant Sciences 2018; 8, 8-14.

[7] Bruto M, Prigent-Combaret C, Muller D, Moënne-Loccoz Y. Analysis of genes contributing to plant-beneficial functions in plant growth-promoting rhizobacteria and related Proteobacteria. Scientific reports 2014; 4, 6261.

[8] Fukao Y, Ferjani A, Tomioka R, Nagasaki N, Kurata R, Nishimori Y, et al. iTRAQ analysis reveals mechanisms of growth defects due to excess zinc in Arabidopsis. Plant Physiology 2011; 155(4), 1893-1907.

[9] Tamura K, Stecher G, Peterson D, Filipski A, Kumar S. MEGA6: molecular evolutionary genetics analysis version 6.0. Molecular biology and evolution 2013; 30(12), 2725-2729.

[10] Saitou N, Nei M. The neighbor-joining method: a new method for reconstructing phylogenetic trees. Molecular biology and evolution 1987; 4(4), 406-425.

[11] Letunic I, Bork P. Interactive tree of life (iTOL) v3: an online tool for the display and annotation of phylogenetic and other trees. Nucleic acids research 2016; 44(W1), W242-W245.

[12] Gerlt JA, Bouvier JT, Davidson DB, Imker HJ, Sadkhin B, Slater $D R$, et al. Enzyme function initiative-enzyme similarity tool (EFI-EST): A web tool for generating protein sequence similarity networks. Biochimica Et Biophysica Acta (BBA)-Proteins and Proteomics 2015; 1854(8), 1019-1037.

[13] Shannon P, Markiel A, Ozier O, Baliga NS, Wang JT, Ramage D, et al. Cytoscape: a software environment for integrated models of biomolecular interaction networks. Genome research 2003; 13(11), 2498-2504.

[14] Tanaka E, Bailey T, Grant CE, Noble WS, Keich U. Improved similarity scores for comparing motifs. Bioinformatics 2011; 27(12), 1603-1609.

[15] Szklarczyk D, Gable AL, Lyon D, Junge A, Wyder S, Huerta-Cepas J, et al. STRING v11: protein-protein association networks with increased coverage, supporting functional discovery in genome-wide experimental datasets. Nucleic acids research 2018; 47(D1), D607-D613.

[16] Singh RP, Runthala A, Khan S, Jha PN. Quantitative proteomics analysis reveals the tolerance of wheat to salt stress in response to Enterobacter cloacae SBP-8. PloS one 2017; 12(9), e0183513.

[17] Saikia J, Sarma RK, Dhandia R, Yadav A, Bharali R, Gupta VK, et al. Alleviation of drought stress in pulse crops with ACC deaminase producing rhizobacteria isolated from acidic soil of Northeast India. Scientific reports 2018; 8(1), 3560.

[18] Souza RD, Ambrosini A, Passaglia LM. Plant growth-promoting bacteria as inoculants in agricultural soils. Genetics and molecular biology 2015; 38(4), 401-419.

[19] Etesami H, Maheshwari DK. Use of plant growth promoting rhizobacteria (PGPRs) with multiple plant growth promoting traits in stress agriculture: Action mechanisms and future prospects. Ecotoxicology and environmental safety 2018; 156, 225-246.

[20] Hanson SR, Best MD, Wong CH. Sulfatases: structure, mechanism, biological activity, inhibition, and synthetic utility. Angewandte Chemie International Edition 2004; 43(43), 5736-5763.

[21] Xavier KB, Miller ST, Lu W, Kim JH, Rabinowitz J, Pelczer I, et al. Phosphorylation and processing of the quorum-sensing molecule autoinducer- 2 in enteric bacteria. ACS chemical biology 2007; 2(2), 128-136.

[22] Zhang Y, Zagnitko O, Rodionova I, Osterman A, Godzik A. The FGGY carbohydrate kinase family: insights into the evolution of functional specificities. PLoS computational biology 2011; 7(12), e1002318.

[23] Enderle J, Dorn A, Beying N, Trapp O, Puchta H. The Protease WSS1A, the Endonuclease MUS81, and the Phosphodiesterase TDP1 Are Involved in Independent Pathways of DNA-protein Crosslink Repair in Plants. The Plant Cell 2019; 31(4), 775-790.

[24] Bionda T, Gross LE, Becker T, Papasotiriou DG, Leisegang MS, Karas M, et al. Eukaryotic Hsp70 chaperones in the intermembrane space of chloroplasts. Planta 2016; 243(3), 733-747.

[25] Ray D, Ghosh A, Mustafi SB, Raha S. Plant stress response: Hsp70 in the spotlight. In Heat Shock Proteins and Plants (pp. 123-147). Springer, Cham.; 2016.

[26] Sterner DE, Berger SL. Acetylation of histones and transcriptionrelated factors. Microbiol. Mol. Biol. Rev. 2000; 64(2), 435-459.

[27] Mwita L, Chan WY, Pretorius T, Lyantagaye SL, Lapa SV, Avdeeva $\mathrm{LV}$, et al. Gene expression regulation in the plant growth promoting Bacillus atrophaeus UCMB-5137 stimulated by maize root exudates. Gene 2016; 590(1), 18-28.

[28] Loose M, Mitchison TJ. The bacterial cell division proteins FtsA and FtsZ self-organize into dynamic cytoskeletal patterns. Nature cell biology 2014; 16(1), 38.

[29] Mayer F. Cytoskeletons in prokaryotes. Cell biology international 2003; 27(5), 429-438.

[30] Deng W, Li C, Xie J. The underling mechanism of bacterial TetR/ AcrR family transcriptional repressors. Cellular signaling 2013; 25(7), 1608-1613.

[31] Reddy VS, Shlykov MA, Castillo R, Sun El, Saier Jr MH.. The major facilitator superfamily (MFS) revisited. The FEBS journal 2012; 279(11), 2022-2035.

[32] Struck AW, Thompson ML, Wong LS, Micklefield J. S-Adenosylmethionine-dependent methyltransferases: highly versatile enzymes in biocatalysis, biosynthesis and other biotechnological applications. ChemBioChem, 2012; 13(18), 2642-2655.

[33] Gao CH, Yang M, He ZG. Characterization of a novel ArsR-like regulator encoded by Rv2034 in Mycobacterium tuberculosis. PLoS One 2012; 7(4), e36255.

[34] Weber IT, Takio K, Titani K, Steitz TA. The cAMP-binding domains of the regulatory subunit of CAMP-dependent protein kinase and the catabolite gene activator protein are homologous. Proceedings of the National Academy of Sciences 1982; 79(24), 7679-7683.

[35] Scheible WR, Pauly M. Glycosyltransferases and cell wall biosynthesis: novel players and insights. Current opinion in plant biology 2004; 7(3), 285-295.

[36] Bhate MP, Molnar KS., Goulian, M., DeGrado, WF. Signal transduction in histidine kinases: insights from new structures. Structure 2015; 23(6), 981-994. 


\section{Appendix}

Table 2. The protein families (Pfam) and their description resulted from analysis on Genome Neighbourhood Networks GNN in Hub-Nodes format from 564 protein sequences resulted in seven node clusters indicated with 86 genes.

\begin{tabular}{|c|c|}
\hline Pfam & Pfam Description \\
\hline \multicolumn{2}{|l|}{ Node 1} \\
\hline $\mathrm{HrcA}$ & HrcA protein $\mathrm{C}$ terminal domain \\
\hline GrpE & Factor GrpE domain \\
\hline \multicolumn{2}{|l|}{ Node 14} \\
\hline Ribonuc_L-PSP & Endoribonuclease L-PSP \\
\hline Amidohydro_1 & Amidohydrolase family \\
\hline Abhydrolase_3 & alpha/beta hydrolase fold \\
\hline Ycel & Ycel-like domain \\
\hline SnoaL_2 & SnoaL-like domain \\
\hline DUF1643 & Protein of unknown function (DUF1643) \\
\hline Flavodoxin_2 & Flavodoxin-like fold \\
\hline Pyr_redox_3 & Pyridine nucleotide-disulphide oxidoreductase \\
\hline Glyco_hydro_3-Glyco_hydro_3_C & Glycosyl hydrolase family $3 \mathrm{~N}$ terminal domain-Glycosyl hydrolase family $3 \mathrm{C}$-terminal domain \\
\hline Pkinase & Protein kinase domain \\
\hline Cupin_1 & Cupin \\
\hline Response_reg-Trans_reg_C & Response regulator receiver domain-Transcriptional regulatory protein, $\mathrm{C}$ terminal \\
\hline Chlorophyllase2 & Chlorophyllase enzyme \\
\hline STAS_2 & STAS domain \\
\hline DNA_ligase_A_M-DNA_ligase_A_C & ATP dependent DNA ligase domain-ATP dependent DNA ligase $C$ terminal region \\
\hline DNA_primase_S & DNA primase small subunit \\
\hline PQQ_2 & PQQ-like domain \\
\hline Spolle & Stage II sporulation protein E (SpollE) \\
\hline HisKA-HATPase_c & $\begin{array}{l}\text { His Kinase A (phospho-acceptor) domain-Histidine kinase-, DNA gyrase B-, and HSP90-like } \\
\text { ATPase }\end{array}$ \\
\hline ABC_tran & $A B C$ transporter \\
\hline ABC2_membrane_2 & $A B C-2$ family transporter protein \\
\hline Peptidase_A24-DiS_P_DiS & Type IV leader peptidase family-Bacterial Peptidase A24 N-terminal domain \\
\hline HisKA-HATPase_c-PAS_4 & $\begin{array}{l}\text { His Kinase A (phospho-acceptor) domain-Histidine kinase-, DNA gyrase B-, and HSP90-like } \\
\text { ATPase-PAS fold }\end{array}$ \\
\hline Cupin_2 & Cupin domain \\
\hline Pkinase-PD40 & Protein kinase domain-WD40-like Beta Propeller Repeat \\
\hline PBP-DUF1028 & Phosphatidylethanolamine-binding protein-Family of unknown function (DUF1028) \\
\hline Mce4_CUP1 & Cholesterol uptake porter CUP1 of Mce4, putative \\
\hline MlaD & MlaD protein \\
\hline MlaD-Mce4_CUP1 & MlaD protein-Cholesterol uptake porter CUP1 of Mce4, putative \\
\hline RelE & RelE toxin of RelE / RelB toxin-antitoxin system \\
\hline VirC1 & VirC1 protein \\
\hline AAA_31 & AAA domain \\
\hline T4SS-DNA_transf & Type IV secretory system Conjugative DNA transfer \\
\hline PRiA4_ORF3 & Plasmid pRiA4b ORF-3-like protein \\
\hline GntR-FCD & Bacterial regulatory proteins, gntR family-FCD domain \\
\hline
\end{tabular}




\begin{tabular}{|c|c|}
\hline Pfam & Pfam Description \\
\hline Transp_cyt_pur & Permease for cytosine/purines, uracil, thiamine, allantoin \\
\hline Resolvase & Resolvase, $\mathrm{N}$ terminal domain \\
\hline DUF4158 & Domain of unknown function (DUF4158) \\
\hline HTH_31 & Helix-turn-helix domain \\
\hline DDE_Tnp_Tn3 & Tn3 transposase DDE domain \\
\hline Flavin_Reduct & Flavin reductase like domain \\
\hline Asp_Glu_race & Asp/Glu/Hydantoin racemase \\
\hline \multicolumn{2}{|l|}{ Node 16} \\
\hline PTS-HPr & PTS HPr component phosphorylation site \\
\hline Glyco_hydro_32N-Glyco_hydro_32C & Glycosyl hydrolases family $32 \mathrm{~N}$-terminal domain-Glycosyl hydrolases family $32 \mathrm{C}$ terminal \\
\hline Hydrolase_3 & haloacid dehalogenase-like hydrolase \\
\hline tRNA-synt_1-Anticodon_1 & tRNA synthetases class I (I, L, M and V)-Anticodon-binding domain of tRNA \\
\hline Peptidase_C26 & Peptidase C26 \\
\hline 23S_rRNA_IVP & $23 S$ rRNA-intervening sequence protein \\
\hline Trans_reg_C & Transcriptional regulatory protein, $\mathrm{C}$ terminal \\
\hline $\begin{array}{l}\text { Phage_integrase-Arm-DNA-bind_4-Phage_ } \\
\text { int_SAM_3 } \\
\text { PTS_EIIB-PTS_EIIC }\end{array}$ & $\begin{array}{l}\text { Phage integrase family-Arm DNA-binding domain-Phage integrase, N-terminal SAM-like } \\
\text { domain } \\
\text { phosphotransferase system, ElIB-Phosphotransferase system, EIIC }\end{array}$ \\
\hline Vsr-DUF559 & DNA mismatch endonuclease Vsr-Protein of unknown function (DUF559) \\
\hline Lacl-Peripla_BP_3 & Bacterial regulatory proteins, lacl family-Periplasmic binding protein-like domain \\
\hline ATP-cone-NRDD & ATP cone domain-Anaerobic ribonucleoside-triphosphate reductase \\
\hline PDDEXK_9-AAA-ATPase_like & PD-(D/E)XK nuclease superfamily-Predicted AAA-ATPase \\
\hline BPD_transp_1 & Binding-protein-dependent transport system inner membrane component \\
\hline Sulfate_transp-STAS & Sulfate permease family-STAS domain \\
\hline DEAD-Helicase_C-HRDC-RecQ_Zn_bind & $\begin{array}{l}\text { DEAD/DEAH box helicase-Helicase conserved C-terminal domain-HRDC domain-RecQ zinc- } \\
\text { binding }\end{array}$ \\
\hline ECF-ribofla_trS & ECF-type riboflavin transporter, S component \\
\hline \multicolumn{2}{|l|}{ Node 19} \\
\hline Plug-CarbopepD_reg_2 & TonB-dependent Receptor Plug Domain-CarboxypepD_reg-like domain \\
\hline Hexapep & Bacterial transferase hexapeptide (six repeats) \\
\hline GSHPx & Glutathione peroxidase \\
\hline MarR & MarR family \\
\hline Pyr_redox_2-Reductase_C & Pyridine nucleotide-disulphide oxidoreductase-Reductase C-terminal \\
\hline OsmC & OsmC-like protein \\
\hline DEDD_Tnp_IS110-Transposase_20 & Transposase-Transposase IS116/IS110/IS902 family \\
\hline Aldo_ket_red & Aldo/keto reductase family \\
\hline \multicolumn{2}{|l|}{ Node 24} \\
\hline VCBS & Repeat domain in Vibrio, Colwellia, Bradyrhizobium and Shewanella \\
\hline Asn_synthase-GATase_7 & Asparagine synthase-Glutamine amidotransferase domain \\
\hline UvrA_DNA-bind-UvrA_inter & UvrA DNA-binding domain-UvrA interaction domain \\
\hline adh_short_C2 & Enoyl-(Acyl carrier protein) reductase \\
\hline E1_dh-Transket_pyr-Transketolase_C & $\begin{array}{l}\text { Dehydrogenase E1 component-Transketolase, pyrimidine binding domain-Transketolase, } \\
\text { C-terminal domain }\end{array}$ \\
\hline Lysine_decarbox & Possible lysine decarboxylase \\
\hline HEM4 & Uroporphyrinogen-III synthase HemD \\
\hline ALAD & Delta-aminolevulinic acid dehydratase \\
\hline
\end{tabular}


Pfam

ACP_syn_III_C-ACP_syn_III

UnbV_ASPIC-VCBS

PseudoU_synth_2

HisKA-HATPase_c-PAS_3-PAS_4

Polysacc deac 1

Glyco_hydro_11

Response_reg-Sigma54_activat-HTH_8

Aminotran_3

AMP-binding-PP-binding
Pfam Description

3-0xoacyl-[acyl-carrier-protein (ACP)] synthase III C terminal -3-0xoacyl-[acyl-carrier-protein (ACP)] synthase III

ASPIC and UnbV-Repeat domain in Vibrio, Colwellia, Bradyrhizobium and Shewanella

RNA pseudouridylate synthase

His Kinase A (phospho-acceptor) domain-Histidine kinase-, DNA gyrase B-, and HSP90-like ATPase-PAS fold-PAS fold

Polysaccharide deacetylase

Glycosyl hydrolases family 11

Response regulator receiver domain-Sigma-54 interaction domain-Bacterial regulatory protein, Fis family

Aminotransferase class-III

AMP-binding enzyme-Phosphopantetheine attachment site 